\title{
Successful Venoplasty of Superior Vena Cava Stenosis in a Patient with a Total Artificial Heart after Orthotopic Heart Transplantation due to Primary Graft Failure
}

\author{
Anita Phancao ${ }^{1}$, Aly El Banayosy ${ }^{2}$, Stephen Lee², David Vanhooser ${ }^{2}$, Michael Harper², \\ Douglas Hortsmanshof ${ }^{2}$, James Long ${ }^{2}$, and Michael M Koerner ${ }^{2}$ \\ ${ }^{1}$ University of Miami Department of Medicine \\ ${ }^{2}$ INTEGRIS Baptist Medical Center
}

May 20, 2020

\begin{abstract}
Background: With the limited number of available suitable donor hearts resulting in plateaued numbers of heart transplantations, short- and long-term mechanical circulatory support devices, including the implantation of total artificial hearts (TAH) are modalities that are increasingly being used as treatment options for patients with end-stage heart failure. The superior vena cava syndrome has been described in this context in various disease processes. We report successful venoplasty for superior vena cava syndrome in a patient with a TAH. Case Presentation: A 65 years old man with a history of non-ischemic cardiomyopathy had received a left ventricular assist device, and then two years later underwent orthotopic heart transplantation using the bicaval anastomosis technique. The post procedural course was complicated by primary graft failure, resulting in the need for the implantation of a TAH. About 5 months after the TAH implantation he started to develop complications such as volume retention, swelling of the upper extremities, and was diagnosed to have a superior vena cava syndrome. The patient underwent a successful venoplasty of his superior vena cava by interventional radiology with resolution of upper body edema, normalization of renal and liver function. Conclusion: Potential fatal complications caused by catheter or wire entrapment in the right sided mechanical valve of a TAH have been reported. We describe a safe method for the treatment of superior vena cava syndrome in patients with TAH.
\end{abstract}

\section{Background}

A total artificial heart (TAH) is a form of heart replacement therapy that provides cardiocirculatory support in end-stage heart failure being used as a bridge to heart transplantation or destination therapy ${ }^{1,2}$. Due to limited availability of acceptable donor hearts, left ventricular assist devices (LVADs), biventricular assist devices, and TAHs are becoming increasingly used as options for the treatment of end-stage heart failure if conventional treatment is failing.

Superior vena cava syndrome (SVCS) is a condition in which the superior vena cava (SVC) is partially blocked or compressed, leading to a constellation of symptoms including upper extremity swelling, shortness of breath, coughing, hoarseness, as well as more serious complications such as cerebral edema, confusion, multi organ dysfunction, and coma ${ }^{3}$.

SVCS is most commonly recognized in patients with malignancies ${ }^{4}$, and has also been reported to be caused by infections ${ }^{5}$, multiple catherization of the venous system, pacemaker placement, and heart transplantation ${ }^{3,6-8}$. Management of SVCS is dependent on the severity of disease as well as underlying etiology. Percutaneous intervention for SVCS is a treatment modality which has provided successful results $^{3}$. Although there have been reports of percutaneous intervention for relief of SVCS in those with 
heart transplantation ${ }^{6,9}$, so far there has only been one case being reported in the literature in regard to the treatment of SVCS in a patient with TAH, although this was caused by external compression of the SVC due to bleeding and tamponade ${ }^{10}$.

\section{Case Presentation}

A 65 year old man with a history of chronic renal insufficiency, dual chamber AICD, non-ischemic cardiomyopathy, and end-stage heart failure received a non-pulsatile LVAD (HeartMate 3®, Abbott, Boston, MA) as a bridge to heart transplantation.

The patient did well while under LVAD support. After two years he was re-evaluated and subsequently listed for heart transplantation. He received an appropriately matched donor heart which was implanted using the bicaval anastomosis technique. Unfortunately his orthotopic heart transplantation was complicated by

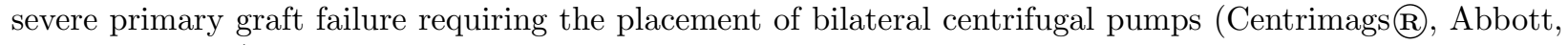
Pleasanton, CA). His donor heart did not recover and therefore he received an implantation of a $70 \mathrm{cc}$ TAH (CardioWest temporary Total Artificial Heart System; SynCardia Systemsß, Inc, Tucson, AZ). He had a prolonged hospitalization during which he required the placement of a temporary dialysis catheter for continuous renal replacement therapy. This procedure was done under continuous fluoroscopy to avoid any life-threatening complications such as entrapment of the guide wire and/or line into the right sided mechanical valve of his TAH. In the following time, his renal function eventually recovered but he chronically required high dosages of diuretics. At that time he also experienced episodes of confusion, agitation, emotional lability, and short episodes of expressive aphasia. The computed tomography of his head only noted a small subarachnoid hemorrhage in the left frontal lobe. The patient recovered and was discharged home.

Three months after TAH implantation he was admitted with shortness of breath, weight gain, low fill volumes and low cardiac output readings on his TAH. Facial and upper extremity swelling was noted. A central line was placed under continuous imaging with projection of its tip just on the upper part of the SVC. The readings of his central venous pressure were exceeding values of $20 \mathrm{~cm}$ of $\mathrm{H}_{2} \mathrm{O}$. Due to significant hypertension he was treated with multiple antihypertensive medications and diuretics.

Subsequently he was readmitted monthly to bimonthly with various problems such as epistaxis and labile mood swings. During all these admissions edema of his upper extremities, primarily left sided edema, and facial swelling had been noted. During his entire post TAH implantation course, he did receive multiple fluoroscopy guided central lines, as well as temporary dialysis catheter placements for volume removal due to diuretic resistance. The tips of the guide wires and lines were, intentionally, never inserted deeper than just into the upper part of the SVC to avoid the possibility of a wire and or a line entrapment into the TAH. A subclavian access was considered as contraindicated due to the risk that lifting his arm may generate the possibility that the tip of the line could advance deep enough getting entrapped in the right sided valve. The central venous pressures were always noted to be significantly elevated and considered to be caused by renal insufficiency in a patient with questionable compliance and intermittent need for intravenous diuretics and/or renal replacement therapy.

Due to the patient's recurrent clinical presentations, an additional component such as SVCS was suspected, although venous ultrasound studies of his upper extremities did not reveal any deep venous thrombosis or occlusion. During one of his admissions for volume retention, a temporary dialysis catheter placement was performed by interventional radiology with findings consistent of severe stenosis of the SVC. The major concern for a possible venoplasty was that the treatment option carried the possibility for the guide wire not to pass immediately through the stenosis and curve above the stenosis without passing through the opening, which could eventually build up kinetic energy during the attempt to correct its position and unintentionally suddenly flip through the stenosis vested with additional kinetic energy and length when straightened. This uncontrolled move may hypothetically deviate the wire into the right sided valve of the TAH rather than to stay in the patient's SVC or inferior vena cava. Under risk benefit analysis and informed consent by the patient and his proxies, the decision was made for venoplasty of the SVC stenosis by interventional radiology under fluoroscopy guidance. The pressure gradient within the SVC was measured above the stenosis at 30 
$\mathrm{mmHg}$ and below the stenosis at $18 \mathrm{mmHg}$ (Figure 1,2). The patient's SVC was serially dilated with 10 $\mathrm{mm}, 14 \mathrm{~mm}$, and $18 \mathrm{~mm}$ diameter angioplasty balloons (Figures 3,4). The $12 \mathrm{mmHg}$ gradient was decreased finally down to $3 \mathrm{mmHg}$ after angioplasty (Figure 5). The patient's symptoms including the swelling of his upper extremities, shortness of breath, and hoarseness all improved post procedure. He has been discharged and will be evaluated for combined heart/kidney transplantation.

\section{Conclusion}

Durable mechanical circulatory support is becoming increasingly used due to the shortage of available or suitable donor hearts for end-stage heart failure. External and intravascular mechanical complications will arise with increased use of these devices, the use of the bicaval anastomosis technique for orthotopic heart transplantation, as well as placement of central venous catheters.

Entrapment of guide wires, catheters or central lines into the right ventricular inflow valves of a TAH is a fatal complication in the management of this patient population ${ }^{11}$. Although stent placement for SVCS caused by external compression due to bleeding and tamponade in TAH has previously been described ${ }^{10}$, the risk of stent migration in endovascular stenting in those with $\mathrm{SVCS}^{9}$ could create lethal outcomes in this population. In life-threatening situations such as profound hypoxic lung dysfunction and adult respiratory distress syndrome after TAH implantation ${ }^{12,13}$ invasive instrumentation with the help of guide wires has been performed successfully. Here we describe a safe and feasible treatment option in TAH patients with hemodynamically relevant SVCS caused by primarily vascular related impairment with venoplasty of the SVC.

The development of SVCS after advanced cardiac therapy therapies, including TAH and heart transplantation may have multiple contributing factors. In patients with TAH after heart transplantation, the development of a SVC stenosis may have a higher incidence due to remaining donor heart tissue that may be a focus of immune related changes with the development of scarring and/or necrosis, especially if immunosuppressive therapy is stopped after the graft has been removed and TAH implanted. Patients with the need of TAH after orthotopic heart transplantation, multiple manipulations due to central line placement in the SVC, defibrillator or pacemaker insertion and manipulations, heart transplant recipients with bicaval rather than biatrial anastomosis between the recipient and the donor heart, all may have a higher tendency to develop SVCS although this remains a topic of clinical observation and discussion.

\section{References}

1. El-Banayosy A, Arusoglu L, Morshuis M, Kizner L, Tenderich G, Sarnowski P, Milting H, Koerfer R: CardioWest total artificial heart: Bad Oeynhausen experience. Ann J Thorac Surg 2005; 80(2):548-52.

2. Copeland JG, Copeland H, Gustafson M, Mineburg N, Covington D, Smith RG, Friedman M: Experience with more than 100 total artificial heart implants. J Thorac Cardiovasc Surg 2012;143:727-34.

3. Wilson LD, Detterbeck F, Yahalom J. Clinical practice. Superior vena cava syndrome with malignant causes. N Engl J Med 2007;356(18):1862-9.

4. Lepper PM, Ott SR, Hope H, Schumann C, Stammberger U Bugalho A, Frese S, Schmucking M, Blumstein NM, Diehm N, Bals R, Jamacher J. Superior Vena Cava Syndrome in Thoracic Malignancies. Respir Care 2011;56(5):653-666.

5. Nunnelee JD. Superior vena cava syndrome. J Vasc Nurs 2007; 25(1): 2-5 quiz 6

6. Strecker T, Zimmerman I, Heinz M, Rosch J, Agaimy A, Weyan M. Successful venous angioplasty of superior vena cava syndrome after heart transplantation. Case Rep Cardiol 2014; 2014:490276.

7. Kashani BS, Ahmadi ZH, Abdi S, Mirhosseini SM, Kianfar AA Niusha S. Superior vena cava obstruction after heart transplantation. Asian Cardiovasc Thorac Ann. 2016;24(1):88-90.

8. Metzer C, Lembcke A, Ziemer S, Eddicks S, Witte J, Baumann G, Borges AC. Pacemaker induced superior 
vena cava syndrome: clinical evaluation of long term follow-up. Pacing Clin Electrophysiol 2006;29(12):134651.

9. Rejjal L, Al-Taweel O, Alwash H, Choudhry M. Alghrouz M, Khalife W, Morsy M, Al-Taweel A, Chatila K, Gilani K, Gilani S. Stent migration during endovascular stenting in superior vena cava syndrome in a patient with recent heart transplantation. JACC 2019;79(9):2741.

10. Kondruweit M, Strecker T, Seitz T, Uder M, Weyand M, Tandler R, Stenting of the superior vena cava in a patient with a total artificial heart. J Thorac Cardiovasc Surg 2008; 135:455-6.

11. Zimmerman H, Coehlo-Anderson R, Slepian M, Smith RG, Sethi G, Copeland JG: Device malfunction of the CardioWest Total Artificial Heart secondary to catheter entrapment of the tricuspid valve. ASAIO J 2010;56:481-82.

12. Spiliopoulos S, Dogan G, Guersoy D, Serrano MR, Koerfer R, Tenderich G: veno-venous extracorporeal membrane oxygenation with a bicaval dual-lumen catheter in a Syncardia total artificial heart patient. J Cardiothorac Surg 2013; 8:179.

13. Lick SD, Tran PL, Kazui P, Smith RG, Khalpey ZI: Total Artificial Heart, augmented by venovenous extracorporeal membrane oxygenation. ASAIO J 2016;62(4): e35-6.

Figure 1: Angiographic catheter (5 French Berenstein, 65 cm length, Torcon NB Beacon Tip, Cook, Inc., Bloomington, IN) inserted from the right common femoral vein through a 9 French vascular sheath. The tip of the catheter is situated in the upper portion of the inferior vena cava prior to passage through the TAH and into the SVC.

Figure 2 : Superior venacavogram using a straight flush catheter (5 French, $65 \mathrm{~cm}$ length, Boston Scientific, Marlborough MA). A high-grade stenosis of the superior vena cava is evident.

Figure 3 : Angioplasty balloon catheter (14 mm diameter Atlas, 7 Fr, Bard Incorporated Tempe, AZ) positioned within the superior vena cava. Balloon is in the process of being inflated and demonstrates the site of vena cava stenosis (full inflation of the balloon not depicted).

Figure 4 : Further angioplasty performed with a larger diameter balloon. Fully inflated $18 \mathrm{~mm}$ diameter angioplasty balloon (Atlas, 7 Fr, Bard Inc., Tempe AZ) across the site of SVC stenosis.

Figure 5 : Repeat superior venacavogram shows no hemodynamically significant residual stenosis.

FIGURE 1 


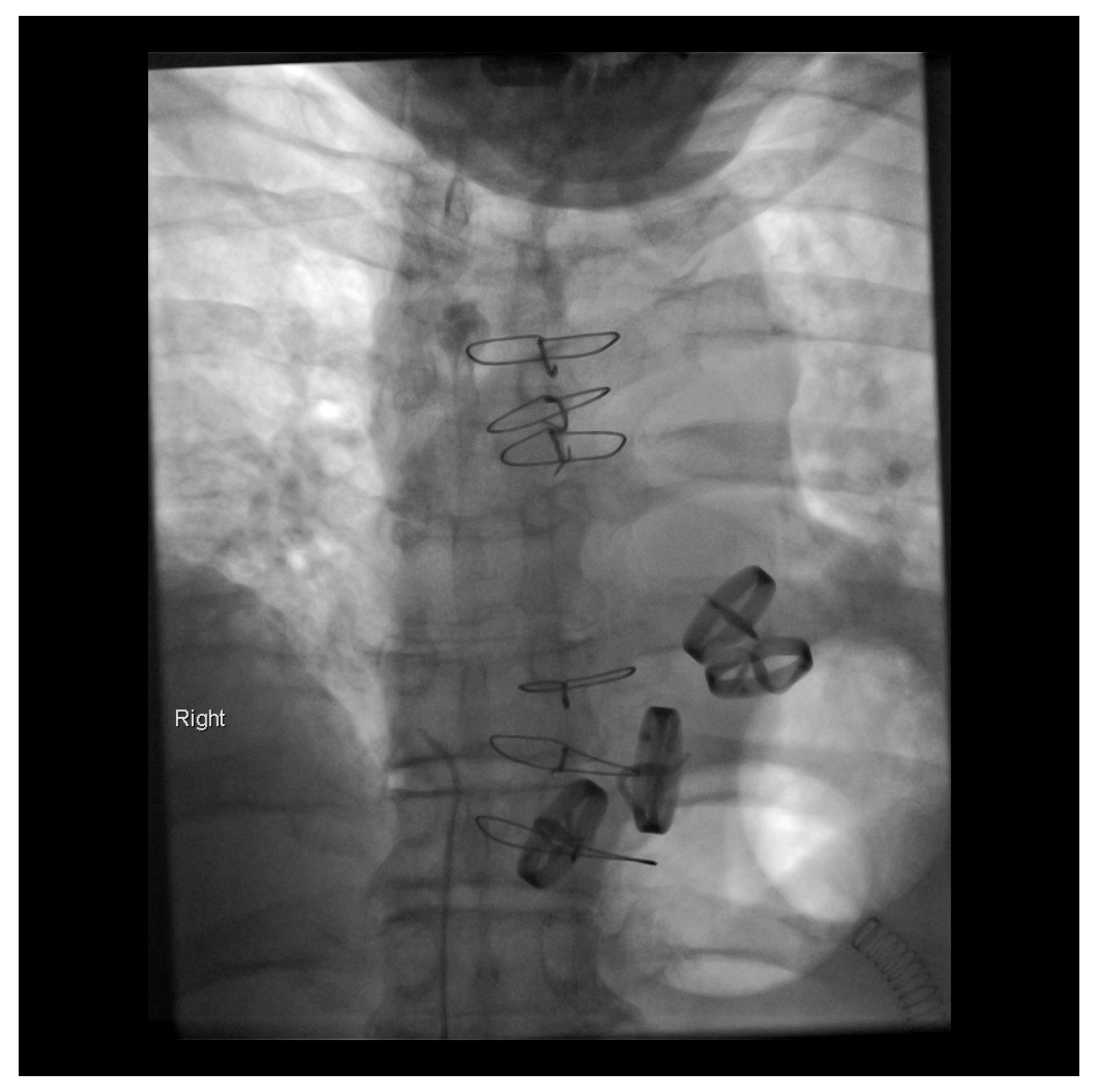

FIGURE 2 


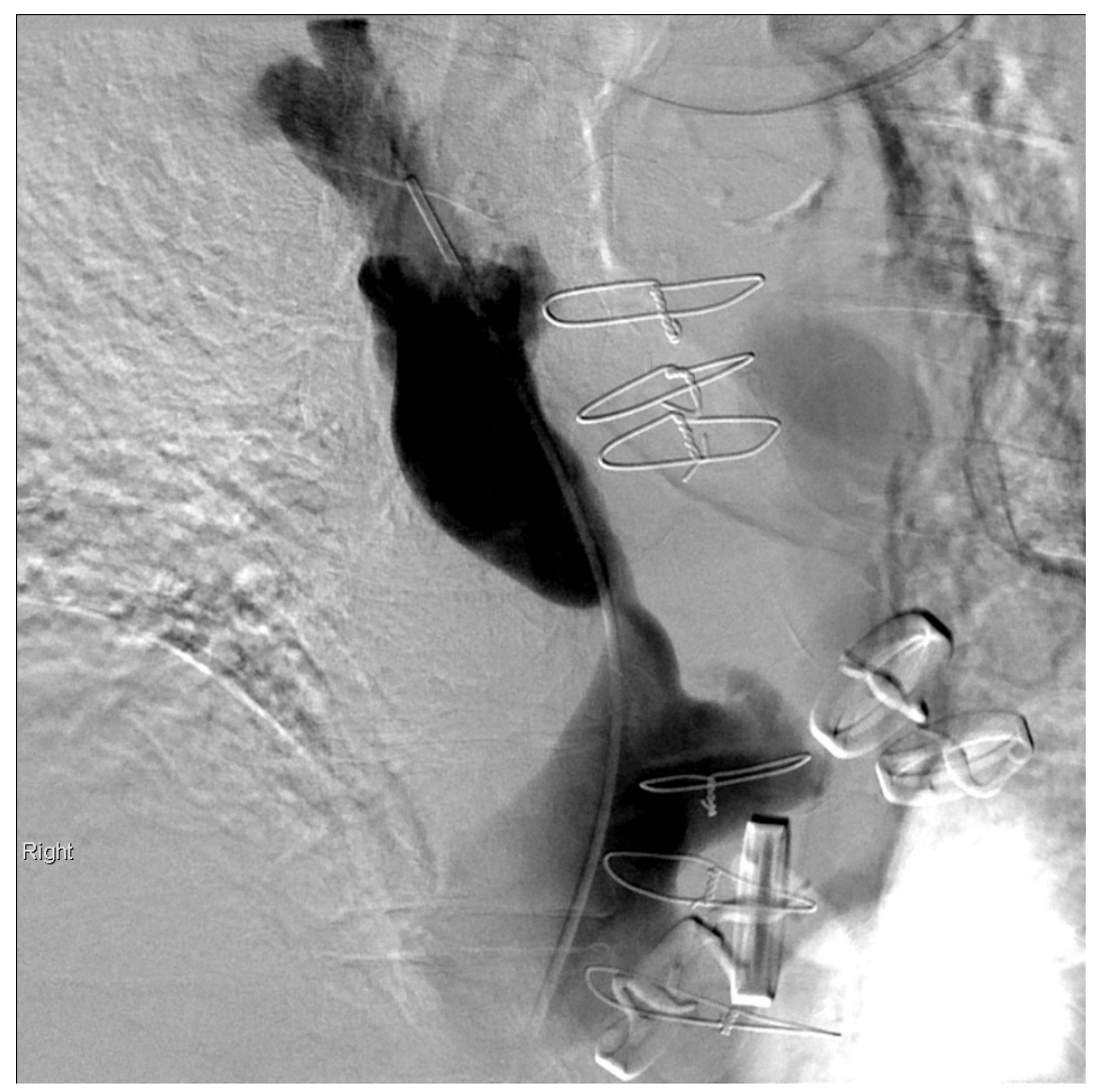

FIGURE 3 


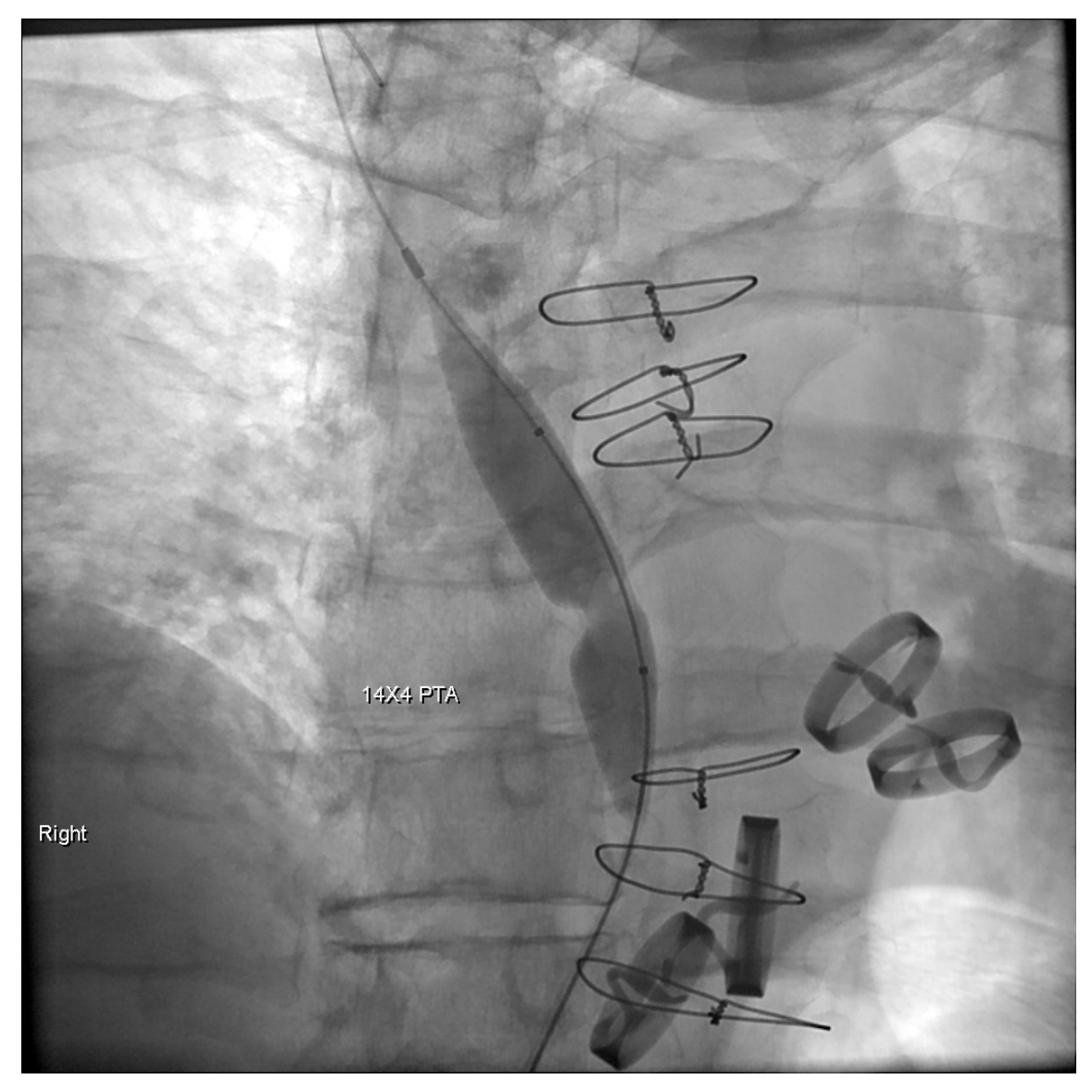

FIGURE 4 


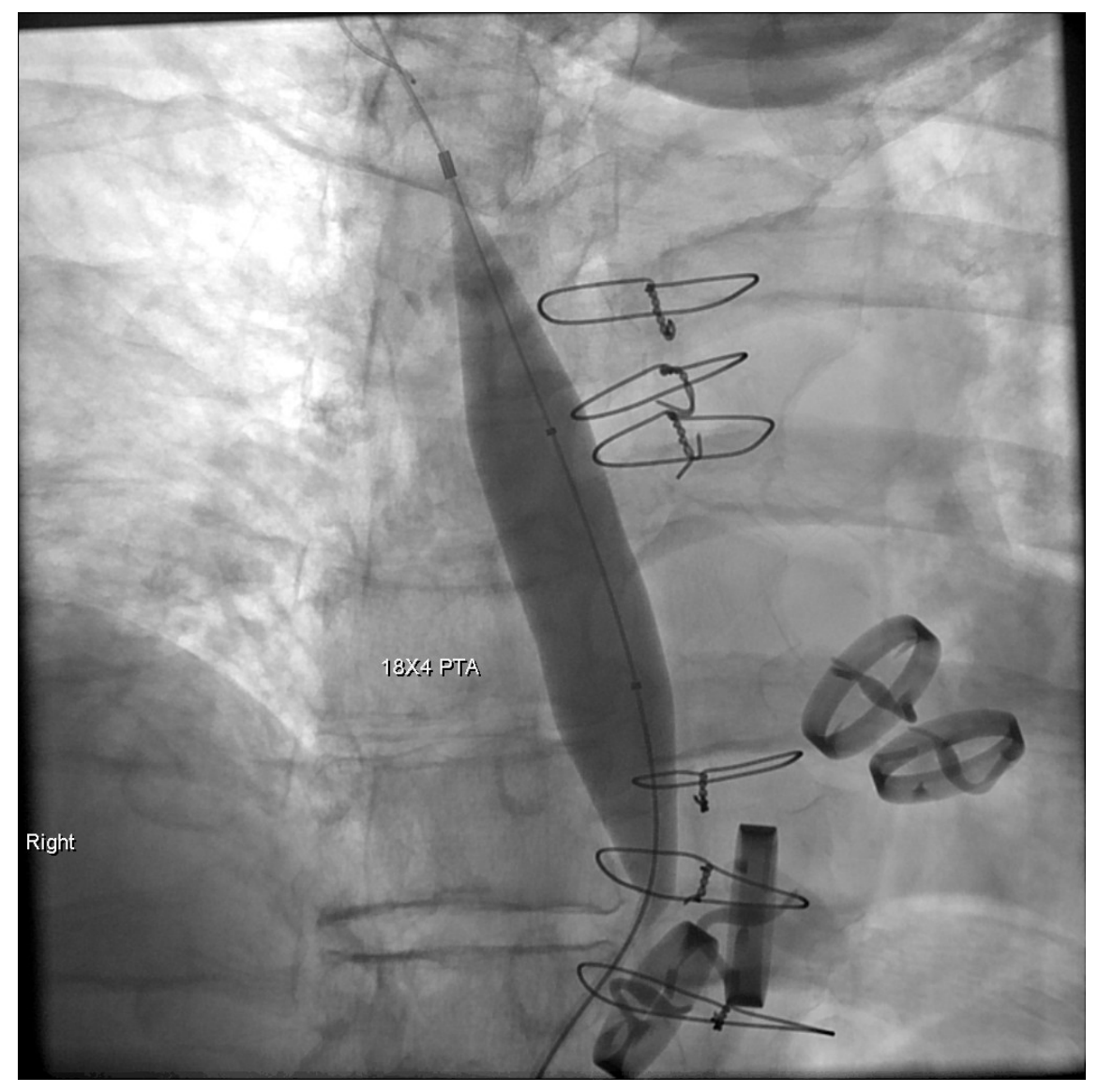

FIGURE 5 


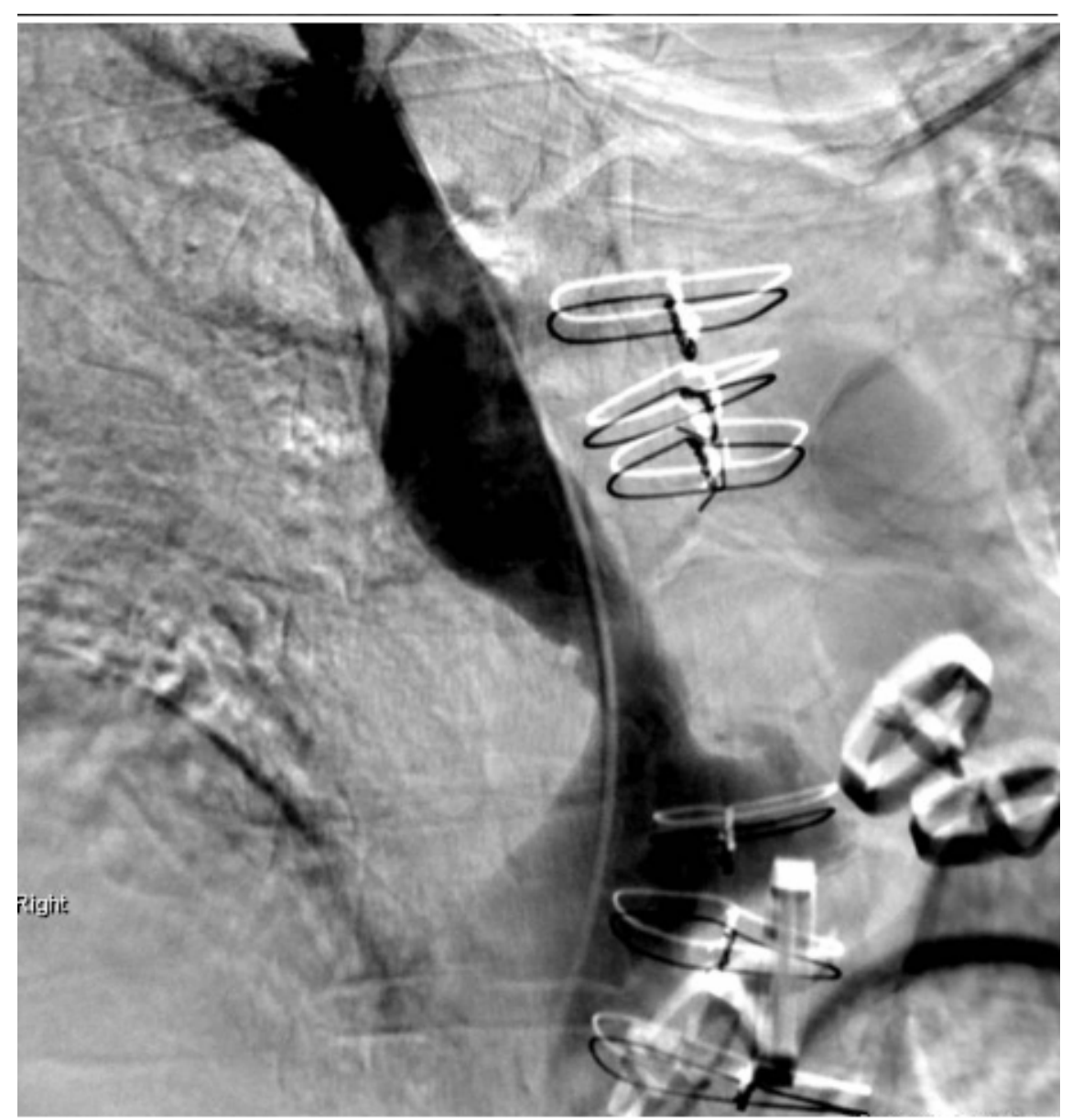

